\title{
Software para evaluación de construcciones dustentables: caso práctico
}

\author{
Eunice Villicaña Ortiz, Ubaldo R. Montes Juárez, Olga E. Serena Ibáñez, \\ Ana G. Pérez Aguilar, Gabriela Martínez Rosiles, María R. Zarate Nava, \\ Jesús L. López Hernández
}

Universidad Tecnológica del Centro de Veracruz,

Av. Universidad No.350, Carretera Federal Cuitláhuac-La Tinaja, Congregación Dos Caminos, Cuitláhuac, Veracruz, C.P. 94910 México

\{soloenergias, ubaldo.montes.juarez, olga.serena.ibanez, anagabrielaperezaguilar, gmrnov92, ma.reina.zarate, jleonardolh\}@gmail.com

\begin{abstract}
From the energy efficiency's point of view in construction, buildings are open thermodynamic systems, where the dull and translucent walls swap energy with the environment, therefore they're not in thermal balance, this state suggest us the necessity to establish mechanisms to know their behavior before the external and internal variations. Because of that, the conception of a building, from the design to the habitability, an energy and comfort analysis should be considered, due to the climatic and geographic conditions of the place where the house is meant to be built, that will allow to identify the thermodynamic behavior and that will anticipate the energy consumption that will demand the system. SICODES is a software that virtually evaluates the behavior of the covering transmittance of a building according to the user specifications and climatic conditions of the place. At the end, SICODES establishes the level of energy efficiency of the construction.
\end{abstract}

Palabras clave. Ingeniería de software, sustentabilidad, evaluación, simulación.

\section{Introducción}

El aumento del consumo energético en las últimas décadas ha impactado en el medio ambiente, se estima que el $75 \%$ de emisiones de Gas de efecto invernadero (GEI) son atribuibles al sector energético [7]. En este sentido, el sector de la construcción ha contribuido de sobremanera, si se consideran los procesos que se realizan desde el diseño, la construcción y habitabilidad de las obras.

Se estima que en promedio una casa para cuatro personas tiene un consumo de 300 $\mathrm{KWh} / \mathrm{mes}$, esto supone un consumo medio anual de 3.6 MWh [2]. Estos datos reflejan un importante consumo para satisfacer las necesidades habituales, no obstante pueden 
establecerse estrategias de ahorro como el cambio de luminaria, el establecimiento de horarios de cargas eléctricas, el correcto dimensionado del sistema eléctrico, etc., que si bien permiten reducir el consumo, por si solas no pueden considerarse como las única alternativas.

La aplicación de la eficiencia energética en edificios antes del diseño es un tema que cobra importancia, y que debe ser atendido en el corto plazo. Determinar la demanda energética que tendrá un edificio permitirá realizar adecuaciones en cuanto a orientación, morfología y materiales a emplear con el fin de reducir el consumo energético dado por la iluminación, calefacción o refrigeración. Por ello el desarrollo de herramientas y técnicas que permitan prever el comportamiento energético de un edifico, es un tema de suma importancia. El desarrollo de edificaciones energéticamente sustentables en México apenas se empieza a trabajar, por lo cual aún no se ha tenido un avance significativo, pues aún se carece de la cultura del cuidado del medio ambiente y la edificación bioclimática. Algunos países han desarrollado herramientas de este tipo, tal es el caso de España, país que integró un documento que contiene las herramientas de cálculo para disminuir la demanda energética a través de técnicas de refrigeración, calefacción, iluminación, diseño de cerramientos, etc. Esta información se encuentra contenida en el Código Técnico de la Edificación, mismo que es utilizado para los programas informáticos CALENER y LIDER los cuales se enfocan en la certificación energética de edificios [2],[3].

El desarrollo de una aplicación que permita simular en entorno 3D la envolvente de un edificio y evaluar su comportamiento a través del análisis de transferencia térmica de sus cerramientos, tales como paredes, techos y suelos, todo ello en función a las condiciones climáticas del lugar donde ha de ser construida, será de gran impacto tecnológico y ambiental en México, dado que no existe un software de simulación y evaluación de construcciones. El presente artículo describe el caso práctico Simulador de Construcciones Energéticamente Sustentables (SICODES).

\section{Aplicación de la metodología}

La metodología empleada para el desarrollo del caso práctico SICODES, Object Modeling Technique (OMT) se basa en el análisis y diseño orientado a objetos. [8]. La gran virtud que aporta OMT, es su carácter de ser abierta (no propietaria), su objetivo es desarrollar un modelo de lo que va a realizar el sistema, el modelo se expresa en términos de objetos y de relaciones entre ellos, muestra un flujo dinámico de control y las transformaciones funcionales.

\subsection{Fase de análisis}

El propósito de la fase radica en entender y modelar el problema en el dominio de la aplicación. 
Para obtener un modelo de dominio que se encarga de mostrar las propiedades más importantes de la problemática identificada, se diseña un conjunto de diagramas (casos de uso, secuencia, estado, clases, flujo del sistema, diccionario de datos) que permitan representar el modelo de dominio. A continuación la Figura 1, ejemplifica la interacción entre el usuario y el sistema.

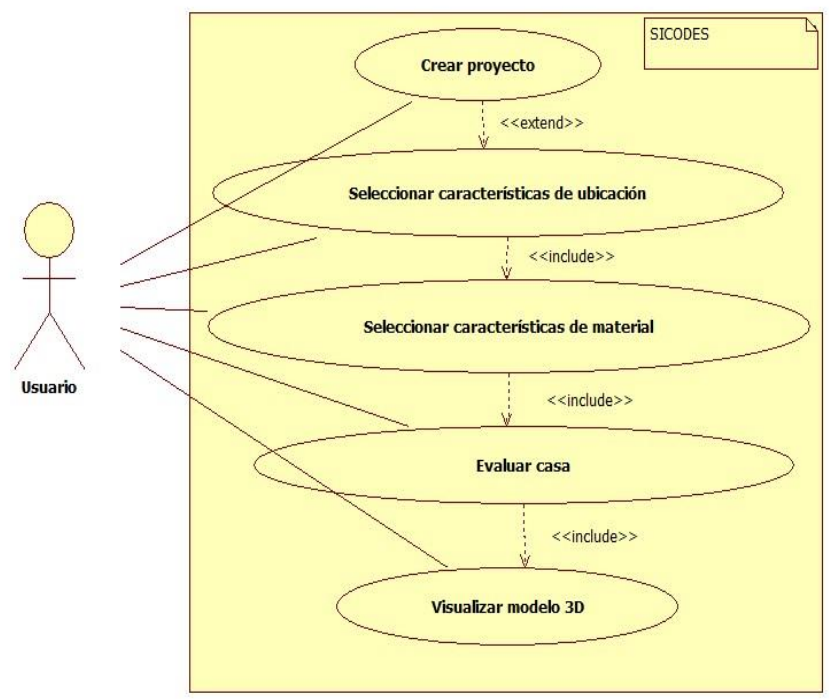

Fig. 1. Diagrama de caso de uso de la aplicación SICODES.

\subsection{Fase de diseño del sistema}

El propósito de la fase radica en definir la arquitectura del sistema y sirve para la tomar decisiones estratégicas. Con base en el análisis de la fase anterior, se diseña una arquitectura software para la implementación en este caso práctico; sirve la arquitectura para examinar la propuesta de construcción de SICODES, estos procesos permiten formular ciertas estrategias y decisiones arquitecturales de apoyo en esta fase, así como en el resto de desarrollo de la aplicación.

\subsubsection{Arquitectura SICODES}

Para el desarrollo, la aplicación basa su implementación en la arquitectura Model-ViewController (MVC), ya que por sus características proporciona escalabilidad, robustez y adaptabilidad a un modelo orientado a objetos, propuesto por OMT. Al ser una 
arquitectura adaptable permite la integración de nuevas capas que admitan la escalabilidad de la misma, la capa evaluación complementa la funcionalidad de esta aplicación.

A continuación se describen las capas que integran la Arquitectura SICODES:

- Vista: Contiene todos los componentes visuales e interfaces de la aplicación, que permiten capturar la información necesaria para efectuar los procesos de diseño. Los materiales 3D que se utilizan para visualizar la edificación son integrados en esta capa.

- Modelo: Las clases que fungen como enlace entre la aplicación y la base de datos se encuentran integradas en esta capa, además de contener las clases que permiten modelar la información relacionada con la zona geográfica, las condiciones climáticas elementales y los materiales que se emplearan en el diseño de la edificación.

- Controlador: Es la capa de comunicación entre la vista y el modelo, es decir que la información ingresada por el usuario es validada y enviada al modelo para hacer actualizaciones a la base de datos de la aplicación. También permite la comunicación con la capa de evaluación, para realizar los cálculos pertinentes en el proceso de evaluación.

- Evaluación: El proceso de evaluación de las características de una edificación es integrado en esta capa, la cual incluye las clases los métodos y fórmulas para evaluar la eficiencia energética, de acuerdo a las características seleccionadas dentro de SICODES.

Las formulas implementadas en esta capa, permiten evaluar con base a las características del espesor y conductividad térmica de los materiales, determinando la resistencia térmica total de la edificación. Además de incorporar en la evaluación factores como: ventanas, puertas y zona geográfica de localización.

Las formulas tomadas para el desarrollo de esta capa, se basan en varios métodos simplificados que se pueden emplear para el cálculo de los parámetros característicos de los diferentes elementos que componen la envolvente térmica de la edificación.[4]. La fórmula 1 muestra la expresión matemática para determinar la resistencia térmica total por pared.

$$
\mathrm{Rt}=\mathrm{Rsi}+\mathrm{R} 1+\mathrm{R} 2+\ldots+\mathrm{Rn}+\mathrm{Rse} .
$$


Una vez calculada la resistencia total de cada pared se procede a determinar la temperatura interior que tendrá la edificación de acuerdo al tipo de cercamientos ingresados por el usuario, pero primero se calcula la temperatura por cada pared de la siguiente manera:

Temperatura por pared $=$ Resistencia total por pared - Temperatura de la ciudad en grados Kelvin.

Una vez que se tiene la temperatura por cada pared, se obtiene un promedio el cual se convierte en la temperatura aproximada que podría llegar a generar una edificación. Ya que se ha obtenido la temperatura promedio de la edificación se procede a determinar la diferencia de temperaturas que existirá entre la temperatura de la ciudad con la de la edificación y en base a este dato se muestra la gráfica indicando si la diferencia de temperaturas es acorde a la zona que el usuario seleccionó para construir la edificación.

La adaptación de la arquitectura MVC y la incorporación de la capa de evaluación permiten la robustez y escalabilidad de SICODES, la cual se expone en la Figura 2.

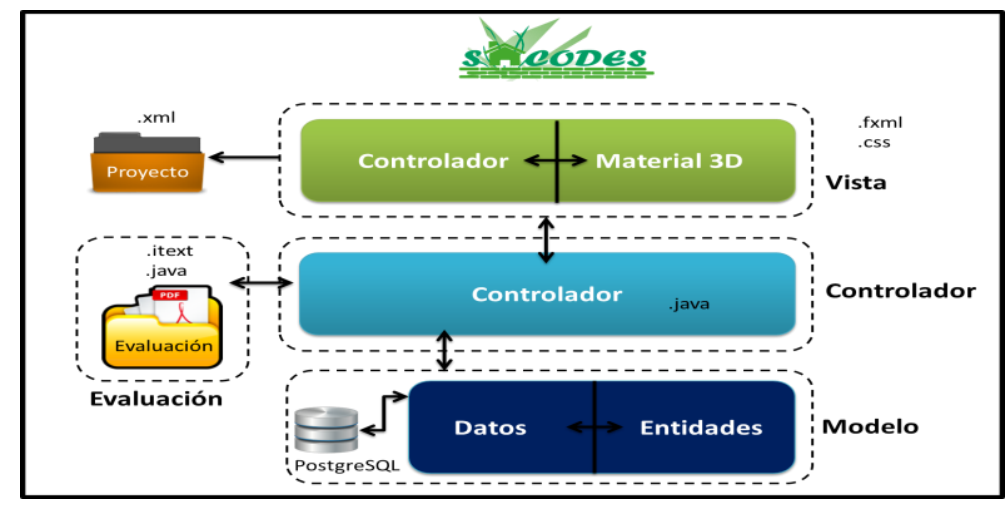

Fig. 2. Arquitectura de SICODES basada en MVC.

\subsection{Fase de diseño de objetos}

El propósito de la fase radica en definir refinar y optimizar el modelo de análisis, agregando conceptos de programación.

Los algoritmos que definen la funcionalidad de la capa de evaluación en la arquitectura SICODES se desarrollaron en esta fase, es decir, se planeó y se diseñó los algoritmos utilizados por la aplicación para el proceso de evaluación de una edificación. Además, se 
buscó la optimización de clases, para que sea más eficiente el modelo que se realizó anteriormente en la fase de análisis. El diseño de objetos se centra en las estructuras de datos y algoritmos que son necesarios para implementar cada clase que se ha planteado ya en fases anteriores.

\subsection{Fase de implementación}

El propósito de la fase radica en definir implementar las clases de objetos en un lenguaje de programación. Empleando la arquitectura SICODES, se generaron 4 paquetes que contienen las clases, modelos y recursos necesarios para la funcionalidad de la aplicación. En la capa vista se modelaron 27 interfaces, 3 hojas de estilo y 48 recursos relacionados con los modelos 3D; en modelo se modelaron 39 clases necesarias para poder realizar conexión a base de datos y se llevó a cabo la implementación del controlador con 22 clases y en la capa de evaluación se tienen las clases clave de la aplicación, en ella se modelaron cada una de las formulas determinantes para la transmitancia térmica.

El resultado final de esta fase de implementación es una gráfica que indica la diferencia de temperaturas entre la zona geográfica y la temperatura que se genera en la edificación, dependiendo de la zona se dan recomendaciones para ajustar los materiales de cómo está constituida la edificación, dicha grafica se muestra en la Figura 3.

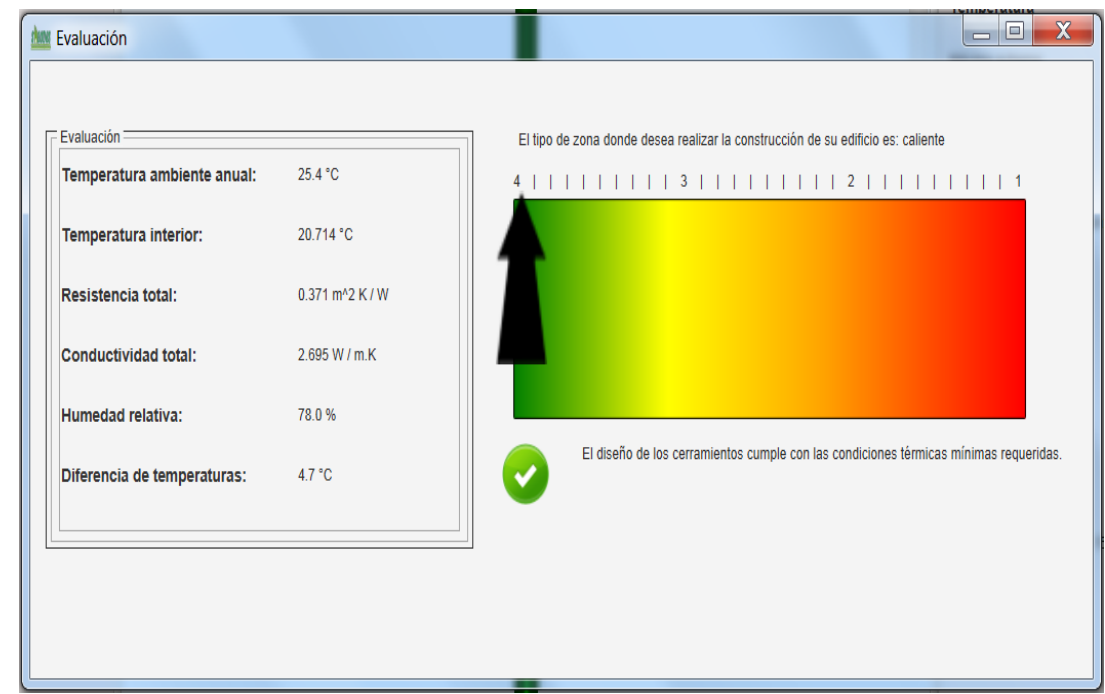

Fig. 3. Gráfica de evaluación de la edificación bajo la aplicación SICODES. 


\section{Resultados}

La implementación de OMT facilito el modelado de los objetos y actores que interactúan en SICODES, este punto fue fundamental para la construcción del mismo. La versión 3.0 de la aplicación permite simular una edificación de uno o dos niveles y cuatro paredes por nivel, además de la selección de los materiales para los cerramientos y la localización geográfica; con esta información se evalúa la transmitancia térmica y se realiza un análisis del confort térmico.

La Figura 4, muestra el proceso básico que se modeló bajo OMT de la simulación y evaluación de una edificación en la aplicación SICODES [4].

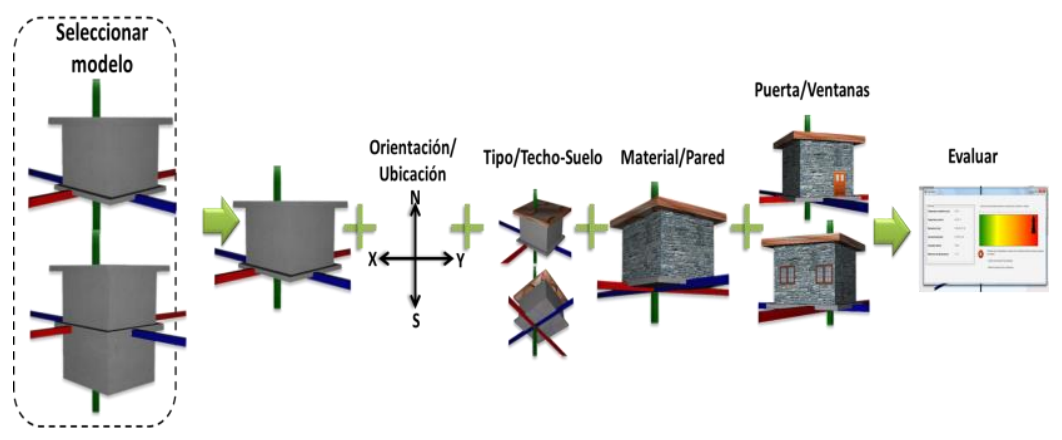

Fig. 4. Flujo básico para el diseño y evaluación de una edificación en SICODES.

Como se observa en la Figura 4, se brinda al usuario la posibilidad de elegir el tipo de edificación que desea simular y personalizarla al seleccionar características como: puertas, ventanas, zona geográfica, material de techo, material del suelo y material de construcción, factores que son importantes para poder determinar el nivel de eficiencia térmica en la evaluación de la edificación.

El uso de la Arquitectura SICODES permitió estructurar los elementos y componentes de la aplicación, brindando la característica de escalabilidad a la aplicación, dada la condición de ampliar su funcionalidad en fases posteriores. Otra de las ventajas, es que el estilo de arquitectura fue dividida en módulos, para implementar la codificación separada y posteriormente ser integrada, facilitando el trabajo en equipo y división de tareas. 


\section{Trabajos relacionados}

\subsection{LIDER}

LIDER es una aplicación informática que se puede emplear para verificar, de forma temporal y bajo ciertas condiciones técnicas, las exigencias de demanda energética establecidas en el Código Técnico de la Edificación de España. Esta herramienta está diseñada para realizar la descripción geométrica, constructiva y operacional de los edificios, así como para llevar a cabo los cálculos de demanda energética de los edificios [2],[3].

\subsection{CALENER VyP}

El programa CALENER VyP (Vivienda y Pequeño edificio terciario) es una de las herramientas informáticas y legislativas que da cuerpo al método general de la Certificación Energética de España. Con ella, se pueden calificar todos los edificios de viviendas, y aquellos edificios del sector terciario, cuyas instalaciones térmicas y de agua caliente sanitaria, se puedan simular con alguno de los modelos que el programa trae adscritos [2],[3].

\subsection{Ecotect}

El software de análisis de diseño sustentable Ecotect es una herramienta de diseño sustentable de edificios que ofrece un amplio rango de simulación y funcionalidad de análisis de energía de las construcciones que puede mejorar el desempeño de los edificios existentes y diseños de nuevos edificios. Capacidades de análisis de energía, agua y emisiones de carbono integradas con herramientas que permiten visualizar y simular el desempeño de un edificio dentro del contexto de su entorno [1].

\subsection{ESP-R}

ESP-R es un programa que permite simular dinámicamente el comportamiento energético de una vivienda o construcción, lo cual permite verificar en fase de diseño las cargas térmicas, tanto de calefacción como de refrigeración. Esto es lo básico que se puede desarrollar con ESP-R ya que también permite el análisis de flujos de aire y aún análisis de CFD (Computer Fluid Dynamic) [5].

\subsection{MIT DESIGN ADVISOR V1.1}

Plataforma Web del MIT que evalúa el comportamiento energético de edificios. Esta herramienta permite evaluar las condiciones de iluminación, ventilación, gasto de energía y confort de un edificio en forma rápida e intuitiva [6]. 


\section{Conclusiones}

La aplicación de OMT para el desarrollo de SICODES ha permitido generar una aplicación que permite la simulación de una edificación en un entorno $3 \mathrm{D}$ y simular el comportamiento de la envolvente de un edificio a partir de las características consideradas por el usuario tales como orientación, climatología, grosor de cerramientos y tamaño de las paredes y material con el cual estará construida. Dentro del equipo de desarrollo la metodología fue fácil de entender e implementar dado que se trabajó con un lenguaje orientado a objetos y gran parte de los artefactos generados son propios del paradigma de programación.

El desarrollo de una aplicación de tal magnitud permitió modelar la Arquitectura SICODES, que facilito el manejo y separación del proyecto en paquetes de trabajo que se traduce en una mejor administración de los recursos para el desarrollo del mismo. SICODES es un proyecto que se encuentra en su tercera fase, la visión es ampliar su funcionalidad para ofrecer mayor impacto en el sector de edificación sustentable en México.

Desde un enfoque sustentable, la construcción debe reducir el impacto medioambiental que produce, es por ello que en los últimos años se ha incrementado el interés por parte de empresas de la industria de la construcción para edificar de forma sustentable. SICODES es una herramienta que permite medir que tan eficiente térmicamente, será una casa antes del proceso de construcción. SICODES busca ser la herramienta que permita a los constructores evaluar y prever el consumo energético de las nuevas edificaciones con el fin de disminuir el consumo energético y el impacto medioambiental en México.

\section{Referencias}

1. Autodesk, http://usa.autodesk.com/ecotect-analysis/, recuperado 18 de Julio (2014)

2. CENEAM. (s.f.). Recuperado el 5 de Julio de 2014, de CENEAM: http://www.magrama.gob.es/ca/ceneam/formacion-ambiental/cursos-ypostgrados/internet/certificacion-energetica-edificios-metodo-lider.aspx, (2014)

3. Codigo tecnico (s.f.) : http://www.codigotecnico.org/web/recursos/aplicaciones/contenido/texto_0002.html, Julio (2014)

4. Documento de Apoyo al Documento Básico DB-HE Ahorro de energía. En M. d. Fomento, Cálculo de parámetros característicos de la envolvente. España.

5. Energy Systems Research Unit. Energy Systems Research Unit. Obtenido de ESP-r: http://www.strath.ac.uk/esru/, (2000)

6. Massachusetts Institute of Technology. MIT Design Advisor. Obtenido de MIT Design Advisor: http://designadvisor.mit.edu/design/, (2009) 
Eunice Villicaña Ortiz, Ubaldo R. Montes Juárez, Olga E. Serena Ibáñez, Ana G. Pérez Aguilar, et al.

7. Trabajo de Investigación: Estudio de la Energía Solar en México. Departamento de Energía, Universidad de Oviedo. España A Survey of Augmented Reality. Presence, 355$385,(2009)$

8. Universidad Nacional Autónoma de México. (n.d.). Metodología OMT. Recuperado el 25 de Julio de 2014 de Posgrado en Ciencia e Ingeniería de la Computación:

http://www.mcc.unam.mx/ cursos/Objetos/Omt/omt.html, (2014) 